\title{
The Explicit Identities for Spectral Norms of Circulant-Type Matrices Involving Binomial Coefficients and Harmonic Numbers
}

\author{
Jianwei Zhou, Xiangyong Chen, and Zhaolin Jiang \\ Department of Mathematics, Linyi University, Shandong 276005, China \\ Correspondence should be addressed to Zhaolin Jiang; jzh1208@sina.com
}

Received 17 October 2013; Accepted 23 December 2013; Published 12 January 2014

Academic Editor: Masoud Hajarian

Copyright (C) 2014 Jianwei Zhou et al. This is an open access article distributed under the Creative Commons Attribution License, which permits unrestricted use, distribution, and reproduction in any medium, provided the original work is properly cited.

\begin{abstract}
The explicit formulae of spectral norms for circulant-type matrices are investigated; the matrices are circulant matrix, skew-circulant matrix, and $g$-circulant matrix, respectively. The entries are products of binomial coefficients with harmonic numbers. Explicit identities for these spectral norms are obtained. Employing these approaches, some numerical tests are listed to verify the results.
\end{abstract}

\section{Introduction}

The classical hypergeometric summation theorems are exploited to derive several striking identities on harmonic numbers [1]. In numerical analysis, circulant matrices (named "premultipliers" in numerical methods) are important because they are diagonalized by a discrete Fourier transform, and hence linear equations that contain them may be quickly solved using a fast Fourier transform. Furthermore, circulant, skew-circulant, and $g$-circulant matrices play important roles in various applications, such as image processing, coding, and engineering model. For more details, please refer to [2-13] and the references therein. The skew-circulant matrices were collected to construct preconditioners for LMF-based ODE codes; Hermitian and skew-Hermitian Toeplitz systems were considered in [1417]; Lyness employed a skew-circulant matrix to construct $s$ dimensional lattice rules in [18]. Recently, there are lots of research on the spectral distribution and norms of circulanttype matrices. In [19], the authors pointed out the processes based on the eigenvalue of circulant-type matrices and the convergence to a Poisson random measure in vague topology. There were discussions about the convergence in probability and distribution of the spectral norm of circulanttype matrices in [20]. The authors in [21] listed the limiting spectral distribution for a class of circulant-type matrices with heavy tailed input sequence. Ngondiep et al. showed that the singular values of $g$-circulants in [22]. Solak established the lower and upper bounds for the spectral norms of circulant matrices with classical Fibonacci and Lucas numbers entries in [23]. İpek investigated an improved estimation for spectral norms in [24].

In this paper, we derive some explicit identities of spectral norms for some circulant-type matrices with product of binomial coefficients with harmonic numbers.

The outline of the paper is as follows. In Section 2, the definitions and preliminary results are listed. In Section 3, the spectral norms of some circulant matrices are studied. In Section 4, the formulae of spectral norms for skew-circulant matrices are established. Section 5 is devoted to investigate the explicit formulae for $g$-circulant matrices. The numerical tests are given in Section 6.

\section{Preliminaries}

The binomial coefficients are defined by $\left(\begin{array}{l}n \\ k\end{array}\right)$ for all natural numbers $k$ at once by

$$
(1+X)^{n}=\sum_{k \geq 0}\left(\begin{array}{l}
n \\
k
\end{array}\right) X^{k}
$$

Note that $\left(\begin{array}{l}n \\ k\end{array}\right)$ is the $k$ th binomial coefficient of $n$. It is clear that $\left(\begin{array}{l}n \\ 0\end{array}\right)=1,\left(\begin{array}{l}n \\ n\end{array}\right)=1$, and $\left(\begin{array}{l}n \\ k\end{array}\right)=0$, for $k>n$. 
The generalized harmonic numbers are defined to be partial sums of the harmonic series [1]:

$$
H_{0}(x)=0, \quad H_{i}(x)=\sum_{k=0}^{i} \frac{1}{x+k} \quad(i=1,2, \ldots) .
$$

For $x=0$ in particular, they reduce to classical harmonic numbers:

$$
H_{0}=0, \quad H_{i}=1+\frac{1}{2}+\frac{1}{3}+\cdots+\frac{1}{i} \quad(i=1,2, \ldots) .
$$

We recall the following harmonic number identities [1]:

$$
\begin{aligned}
& \sum_{i=0}^{n}\left(\begin{array}{c}
n \\
i
\end{array}\right)^{2}\left(\begin{array}{c}
2 n+i \\
i
\end{array}\right)\left(H_{2 n+i}-H_{i}\right) \\
& =2\left(\begin{array}{c}
2 n \\
n
\end{array}\right)^{2}\left(H_{2 n}-H_{n}\right), \\
& \sum_{i=0}^{n}\left(\begin{array}{c}
n \\
i
\end{array}\right)\left(\begin{array}{c}
2 n \\
i
\end{array}\right)\left(\begin{array}{c}
3 n+i \\
i
\end{array}\right)\left(H_{3 n+i}-H_{i}\right) \\
& =\left(\begin{array}{c}
3 n \\
n
\end{array}\right)^{2}\left(2 H_{3 n}-H_{2 n}-H_{n}\right) .
\end{aligned}
$$

Definition 1 (see $[6,8]$ ). A circulant matrix is an $n \times n$ complex matrix with the following form:

$$
A_{c}=\left(\begin{array}{cccc}
a_{0} & a_{1} & \cdots & a_{n-1} \\
a_{n-1} & a_{0} & \cdots & a_{n-2} \\
a_{n-2} & a_{n-1} & \cdots & a_{n-3} \\
\vdots & \vdots & \ddots & \vdots \\
a_{1} & a_{2} & \cdots & a_{0}
\end{array}\right)_{n \times n} .
$$

The first row of $A_{c}$ is $\left(a_{0}, a_{1}, \ldots, a_{n-1}\right)$; its $(j+1)$ th row is obtained by giving its $j$ th row a right circular shift by one position.

Equivalently, a circulant matrix can be described with polynomial as

$$
A_{c}=f\left(\eta_{c}\right)=\sum_{i=0}^{n-1} a_{i} \eta_{c}^{i}
$$

where

$$
\eta_{c}=\left(\begin{array}{ccccc}
0 & 1 & 0 & \cdots & 0 \\
0 & 0 & 1 & \cdots & 0 \\
\vdots & \vdots & \ddots & \vdots & \\
0 & 0 & 0 & \cdots & 1 \\
1 & 0 & 0 & \cdots & 0
\end{array}\right)_{n \times n}
$$

Obviously, $\eta_{c}^{n}=I_{n}$.

Now, we discuss the eigenvalues of $A_{c}$. We declare that the eigenvalues of $\eta_{c}$ are the corresponding eigenvalues of $A_{c}$ with the function $f$ in (6), which is

$$
\lambda\left(A_{c}\right)=f\left(\lambda\left(\eta_{c}\right)\right)=\sum_{i=0}^{n-1} a_{i} \lambda\left(\eta_{c}\right)^{i} .
$$

Whereas $\lambda_{j}\left(\eta_{c}\right)=\omega^{j},(j=0,1, \ldots, n-1)$, then $\lambda_{j}\left(A_{c}\right)$ can be calculated by

$$
\lambda_{j}\left(A_{c}\right)=\sum_{i=0}^{n-1} a_{i}\left(\omega^{j}\right)^{i}
$$

where $\omega=\cos (2 \pi / n)+i \sin (2 \pi / n)$.

Similarly, we recall a skew-circulant matrix.

Definition 2 (see $[6,8])$. A skew-circulant matrix is an $n \times n$ complex matrix with the following form:

$$
A_{\mathrm{sc}}=\left(\begin{array}{cccc}
a_{0} & a_{1} & \cdots & a_{n-1} \\
-a_{n-1} & a_{0} & \cdots & a_{n-2} \\
-a_{n-2} & -a_{n-1} & \cdots & a_{n-3} \\
\vdots & \vdots & \ddots & \vdots \\
-a_{1} & -a_{2} & \cdots & a_{0}
\end{array}\right)_{n \times n} .
$$

Moreover, a skew-circulant matrix can be described with polynomial as

$$
A_{\mathrm{sc}}=f\left(\eta_{\mathrm{sc}}\right)=\sum_{i=0}^{n-1} a_{i} \eta_{\mathrm{sc}}^{i}
$$

where

$$
\eta_{s c}=\left(\begin{array}{ccccc}
0 & 1 & 0 & \cdots & 0 \\
0 & 0 & 1 & \cdots & 0 \\
\vdots & \vdots & \ddots & \vdots & \\
0 & 0 & 0 & \cdots & 1 \\
-1 & 0 & 0 & \cdots & 0
\end{array}\right)_{n \times n} .
$$

Obviously, $\eta_{\mathrm{sc}}^{n}=-I_{n}$.

Thus we have to calculate the eigenvalues of $A_{\mathrm{sc}}$. For the same reason, we obtain that

$$
\lambda\left(A_{\mathrm{sc}}\right)=f\left(\lambda\left(\eta_{\mathrm{sc}}\right)\right)=\sum_{i=0}^{n-1} a_{i} \lambda^{i}\left(\eta_{\mathrm{sc}}\right) .
$$

Whereas $\lambda_{j}\left(\eta_{\mathrm{sc}}\right)=\omega^{j} \alpha,(j=0,1, \ldots, n-1) \lambda_{j}\left(A_{\mathrm{sc}}\right)$ can be computed by

$$
\lambda_{j}\left(A_{\mathrm{sc}}\right)=\sum_{i=0}^{n-1} a_{i}\left(\omega^{j} \alpha\right)^{i}
$$

where $\omega=\cos (2 \pi / n)+i \sin (2 \pi / n), \alpha=\cos (\pi / n)+i \sin (\pi / n)$.

Definition 3 (see $[21,25]$ ). A $g$-circulant matrix is an $n \times n$ complex matrix with the following form:

$$
A_{g}=\left(\begin{array}{cccc}
a_{0} & a_{1} & \ldots & a_{n-1} \\
a_{n-g} & a_{n-g+1} & \ldots & a_{n-g-1} \\
a_{n-2 g} & a_{n-2 g+1} & \ldots & a_{n-2 g-1} \\
\vdots & \vdots & \ddots & \vdots \\
a_{g} & a_{g+1} & \ldots & a_{g-1}
\end{array}\right)_{n \times n},
$$

where $g$ is a nonnegative integer and each of the subscripts is understood to be reduced modulo $n$. 
The first row of $A_{g}$ is $\left(a_{0}, a_{1}, \ldots, a_{n-1}\right)$; its $(j+1)$ th row is obtained by giving its $j$ th row a right circular shift by $g$ positions (equivalently, $g \bmod n$ positions). Note that $g=1$ or $g=n+1$ yields the standard circulant matrix. If $g=n-1$, then we obtain the so-called reverse circulant matrix [21].

Definition 4 (see [26]). The spectral norm $\|\cdot\|_{2}$ of a matrix $A$ with complex entries is the square root of the largest eigenvalue of the positive semidefinite matrix $A^{*} A$ :

$$
\|A\|_{2}=\sqrt{\lambda_{\max }\left(A^{*} A\right)}
$$

where $A^{*}$ denotes the conjugate transpose of $A$. Therefore if $A$ is an $n \times n$ real symmetric matrix or $A$ is a normal matrix, then

$$
\|A\|_{2}=\max _{1 \leq i \leq n}\left|\lambda_{i}\right|,
$$

where $\lambda_{1}, \lambda_{2}, \ldots, \lambda_{n}$ are the eigenvalues of $A$.

\section{Spectral Norms of Some Circulant Matrices}

Now, we will analyse spectral norms of some given circulant matrices, whose entries are binomial coefficients combined with harmonic numbers.

Our main results for those matrices are stated as follows.

Theorem 5. Let $(n+1) \times(n+1)$-circulant matrix $B_{1}$ is as in (5), and the first row of $B_{1}$ is

$$
\begin{aligned}
& \left(\left(\begin{array}{l}
n \\
0
\end{array}\right)^{2}\left(\begin{array}{c}
2 n \\
0
\end{array}\right)\left(H_{2 n}-H_{0}\right),\right. \\
& \left(\begin{array}{c}
n \\
1
\end{array}\right)^{2}\left(\begin{array}{c}
2 n+1 \\
1
\end{array}\right)\left(H_{2 n+1}-H_{1}\right), \ldots \\
& \left.\left(\begin{array}{l}
n \\
n
\end{array}\right)^{2}\left(\begin{array}{c}
2 n+n \\
n
\end{array}\right)\left(H_{2 n+n}-H_{n}\right)\right),
\end{aligned}
$$

where $a_{i}=\left(\begin{array}{c}n \\ i\end{array}\right)^{2}\left(\begin{array}{c}2 n+i \\ i\end{array}\right)\left(H_{2 n+i}-H_{i}\right)$. Then one has

$$
\left\|B_{1}\right\|_{2}=2\left(\begin{array}{c}
2 n \\
n
\end{array}\right)^{2}\left(H_{2 n}-H_{n}\right) \text {. }
$$

Proof. Since circulant matrix $B_{1}$ is normal, employing Definition 4 , we claim that the spectral norm of $B_{1}$ is equal to its spectral radius. Furthermore, applying the irreducible and entrywise nonnegative properties, we claim that $\left\|B_{1}\right\|_{2}$ (i.e., its spectral norm) is equal to its Perron value. We select an $(n+1)$-dimensional column vector $v=(1,1, \ldots, 1)^{T}$; then

$$
B_{1} v=\left(\sum_{i=0}^{n}\left(\begin{array}{c}
n \\
i
\end{array}\right)^{2}\left(\begin{array}{c}
2 n+i \\
i
\end{array}\right)\left(H_{2 n+i}-H_{i}\right)\right) v .
$$

Obviously, $\sum_{i=0}^{n}\left(\begin{array}{c}n \\ i\end{array}\right)^{2}\left(\begin{array}{c}2 n+i \\ i\end{array}\right)\left(H_{2 n+i}-H_{i}\right)$ is an eigenvalue of $B_{1}$ associated with $v$, which is necessarily the Perron value of $B_{1}$. Employing (4), we obtain

$$
\left\|B_{1}\right\|_{2}=2\left(\begin{array}{c}
2 n \\
n
\end{array}\right)^{2}\left(H_{2 n}-H_{n}\right) .
$$

This completes the proof.
Hence, employing the same approaches, we get the following corollary.

Corollary 6. Let $(n+1) \times(n+1)$-circulant matrix $B_{2}$ be as in (5), and the first row of $B_{2}$ is

$$
\begin{aligned}
& \left(\left(\begin{array}{c}
n \\
0
\end{array}\right)\left(\begin{array}{c}
2 n \\
0
\end{array}\right)\left(\begin{array}{c}
3 n \\
0
\end{array}\right)\left(H_{3 n}-H_{0}\right),\right. \\
& \left(\begin{array}{c}
n \\
1
\end{array}\right)\left(\begin{array}{c}
2 n \\
1
\end{array}\right)\left(\begin{array}{c}
3 n+1 \\
1
\end{array}\right)\left(H_{3 n+1}-H_{1}\right), \ldots \\
& \left.\left(\begin{array}{c}
n \\
n
\end{array}\right)\left(\begin{array}{c}
2 n \\
n
\end{array}\right)\left(\begin{array}{c}
4 n \\
n
\end{array}\right)\left(H_{4 n}-H_{n}\right)\right),
\end{aligned}
$$

where $a_{i}=\left(\begin{array}{c}n \\ i\end{array}\right)\left(\begin{array}{c}2 n \\ i\end{array}\right)\left(\begin{array}{c}3 n+i \\ i\end{array}\right)\left(H_{3 n+i}-H_{i}\right)$. Then

$$
\left\|B_{2}\right\|_{2}=\left(\begin{array}{c}
3 n \\
n
\end{array}\right)^{2}\left(2 H_{3 n}-H_{2 n}-H_{n}\right) .
$$

Now, we investigate some even-order alternative as follows, where $m$ is odd (i.e., $m+1$ is even).

Theorem 7. Let $(m+1) \times(m+1)$-circulant matrix $B_{3}$ be as in (5), and the first row of $B_{3}$ is

$$
\begin{aligned}
& \left(\left(\begin{array}{c}
m \\
0
\end{array}\right)^{2}\left(\begin{array}{c}
2 m \\
0
\end{array}\right)\left(H_{2 m}-H_{0}\right),\right. \\
& -\left(\begin{array}{c}
m \\
1
\end{array}\right)^{2}\left(\begin{array}{c}
2 m+1 \\
1
\end{array}\right)\left(H_{2 m+1}-H_{1}\right), \ldots \\
& \left.\quad-\left(\begin{array}{c}
m \\
m
\end{array}\right)^{2}\left(\begin{array}{c}
2 m+m \\
m
\end{array}\right)\left(H_{2 m+m}-H_{m}\right)\right),
\end{aligned}
$$

where $a_{i}=(-1)^{i}\left(\begin{array}{c}m \\ i\end{array}\right)^{2}\left(\begin{array}{c}2 m+i \\ i\end{array}\right)\left(H_{2 m+i}-H_{i}\right)$. Then there holds the following identity:

$$
\left\|B_{3}\right\|_{2}=2\left(\begin{array}{c}
2 m \\
m
\end{array}\right)^{2}\left(H_{2 m}-H_{m}\right) .
$$

Proof. Noticing (9) and (17), it is clear that the spectral norm of $B_{3}$ can be calculated by

$$
\begin{aligned}
\left\|B_{3}\right\|_{2} & =\max _{0 \leq t \leq m}\left|\lambda_{t}\left(B_{3}\right)\right|=\max _{0 \leq t \leq m}\left|\sum_{i=0}^{m} a_{i}\left(\omega^{t}\right)^{i}\right| \\
& \leq \max _{0 \leq t \leq m}\left\{\sum_{i=0}^{m}\left|a_{i}\right| \cdot\left|\left(\omega^{t}\right)^{i}\right|\right\}=\sum_{i=0}^{m}\left|a_{i}\right|,
\end{aligned}
$$

where $a_{i}=(-1)^{i}\left({ }_{i}^{m}\right)^{2}(\underset{i}{2 m+i})\left(H_{2 m+i}-H_{i}\right)$, and we employed that all circulant matrices are normal.

Note that, if $m$ is odd, then $m+1$ is even, and $\lambda_{t_{0}}\left(\eta_{c}\right)=$ $\omega^{t_{0}}=-1$ is an eigenvalue of $\eta_{c}$, so

$$
\left\|B_{3}\right\|_{2}=\sum_{i=0}^{m}\left|a_{i}\right|
$$

Combining (4) and (27) yields

$$
\left\|B_{3}\right\|_{2}=2\left(\begin{array}{c}
2 m \\
m
\end{array}\right)^{2}\left(H_{2 m}-H_{m}\right) \text {. }
$$

This completes the proof. 
Employing the same approaches, we get the following corollary.

Corollary 8. Let $(m+1) \times(m+1)$-circulant matrix $B_{4}$ be as in (5), and the first row of $B_{4}$ is

$$
\begin{aligned}
& \left(\left(\begin{array}{c}
m \\
0
\end{array}\right)\left(\begin{array}{c}
2 m \\
0
\end{array}\right)\left(\begin{array}{c}
3 m \\
0
\end{array}\right)\left(H_{3 m}-H_{0}\right),\right. \\
& -\left(\begin{array}{c}
m \\
1
\end{array}\right)\left(\begin{array}{c}
2 m \\
1
\end{array}\right)\left(\begin{array}{c}
3 m+1 \\
1
\end{array}\right)\left(H_{3 m+1}-H_{1}\right), \ldots, \\
& \left.\left(\begin{array}{c}
m \\
m
\end{array}\right)\left(\begin{array}{c}
2 m \\
m
\end{array}\right)\left(\begin{array}{c}
4 m \\
m
\end{array}\right)\left(H_{4 m}-H_{m}\right)\right)
\end{aligned}
$$

where $a_{i}=(-1)^{i}\left(\begin{array}{c}m \\ i\end{array}\right)\left(\begin{array}{c}2 m \\ i\end{array}\right)\left(\begin{array}{c}3 m+i \\ i\end{array}\right)\left(H_{3 m+i}-H_{i}\right)$. Then one has the following identity:

$$
\left\|B_{4}\right\|_{2}=\left(\begin{array}{c}
3 m \\
m
\end{array}\right)^{2}\left(2 H_{3 m}-H_{2 m}-H_{m}\right) .
$$

Similarly, we set $\widetilde{B}_{3}=-B_{3}, \widetilde{B}_{4}=-B_{4}$.

Corollary 9. Let $\widetilde{B}_{3}, \widetilde{B}_{4}$ be as above, respectively, and $m$ is odd. Then

$$
\begin{gathered}
\left\|\widetilde{B}_{3}\right\|_{2}=2\left(\begin{array}{c}
2 m \\
m
\end{array}\right)^{2}\left(H_{2 m}-H_{m}\right), \\
\left\|\widetilde{B}_{4}\right\|_{2}=\left(\begin{array}{c}
3 m \\
m
\end{array}\right)^{2}\left(2 H_{3 m}-H_{2 m}-H_{m}\right) .
\end{gathered}
$$

\section{Spectral Norms of Skew-Circulant Matrices}

An odd-order alternative skew-circulant matrix is defined as follows, where $s$ is even.

Theorem 10. Let $(s+1) \times(s+1)$-circulant matrix $B_{5}$ be as in (10), and the first row of $B_{5}$ is

$$
\begin{aligned}
& \left(\left(\begin{array}{l}
s \\
0
\end{array}\right)^{2}\left(\begin{array}{c}
2 s \\
0
\end{array}\right)\left(H_{2 s}-H_{0}\right)\right. \\
& \quad-\left(\begin{array}{c}
s \\
1
\end{array}\right)^{2}\left(\begin{array}{c}
2 s+1 \\
1
\end{array}\right)\left(H_{2 s+1}-H_{1}\right), \ldots \\
& \left.\left(\begin{array}{c}
s \\
s
\end{array}\right)^{2}\left(\begin{array}{c}
2 s+s \\
s
\end{array}\right)\left(H_{2 s+s}-H_{s}\right)\right)
\end{aligned}
$$

where $a_{i}=(-1)^{i}\left(\begin{array}{c}s \\ i\end{array}\right)^{2}\left(\begin{array}{c}2 s+i \\ i\end{array}\right)\left(H_{2 s+i}-H_{i}\right)$. Then one obtains

$$
\left\|B_{5}\right\|_{2}=2\left(\begin{array}{c}
2 s \\
s
\end{array}\right)^{2}\left(H_{2 s}-H_{s}\right)
$$

Proof. We employ (14) and (17) to calculate the spectral norm of $B_{5}$ as follows, for all $t=0,1, \ldots, s$ :

$$
\begin{aligned}
\left|\lambda_{t}\left(B_{5}\right)\right| & =\left|\sum_{i=0}^{s} a_{i}\left(\omega^{t} \alpha\right)^{i}\right| \leq \sum_{i=0}^{s}\left|a_{i}\right| \cdot\left|\left(\omega^{t} \alpha\right)^{i}\right| \\
& =\sum_{i=0}^{s}\left|a_{i}\right|=\sum_{i=0}^{s}\left(\begin{array}{c}
s \\
i
\end{array}\right)^{2}\left(\begin{array}{c}
2 s+i \\
i
\end{array}\right)\left(H_{2 s+i}-H_{i}\right),
\end{aligned}
$$

where $a_{i}=(-1)^{i}\left(\begin{array}{c}s \\ i\end{array}\right)^{2}\left(\begin{array}{c}2 s+i \\ i\end{array}\right)\left(H_{2 s+i}-H_{i}\right)$.

Since the skew-circulant matrix is normal, we deduce that

$$
\left\|B_{5}\right\|_{2}=\max _{0 \leq t \leq s}\left|\lambda_{t}\left(B_{5}\right)\right| \text {. }
$$

If $s$ is even, then $s+1$ is odd. We declare that $\lambda_{\mathrm{sc}}=-1$ is an eigenvalue of $\eta_{\mathrm{sc}}$; then we calculate the corresponding eigenvalue of $B_{5}$ as follows:

$$
\begin{aligned}
\lambda_{\hat{t}}\left(B_{5}\right) & =\sum_{i=0}^{s} a_{i} \lambda_{\mathrm{sc}}^{i}=\sum_{i=0}^{s} a_{i}(-1)^{i} \\
& =\sum_{i=0}^{s}\left(\begin{array}{l}
s \\
i
\end{array}\right)^{2}\left(\begin{array}{c}
2 s+i \\
i
\end{array}\right)\left(H_{2 s+i}-H_{i}\right)
\end{aligned}
$$

where we had employed (14).

Noticing (34), we claim that $\lambda_{\widehat{t}}\left(B_{5}\right)$ is the maximum of $\left|\lambda_{t}\left(B_{5}\right)\right|$, which means

$$
\left\|B_{5}\right\|_{2}=\sum_{i=0}^{s}\left(\begin{array}{l}
s \\
i
\end{array}\right)^{2}\left(\begin{array}{c}
2 s+i \\
i
\end{array}\right)\left(H_{2 s+i}-H_{i}\right) .
$$

Thus, from (4) we obtain

$$
\left\|B_{5}\right\|_{2}=2\left(\begin{array}{c}
2 s \\
s
\end{array}\right)^{2}\left(H_{2 s}-H_{s}\right) .
$$

This completes the proof.

Similarly, we can calculate the identity for $B_{6}$.

Corollary 11. Let $(s+1) \times(s+1)$-circulant matrix $B_{6}$ be as in (10), and the first row of $B_{6}$ is

$$
\begin{aligned}
& \left(\left(\begin{array}{l}
s \\
0
\end{array}\right)\left(\begin{array}{c}
2 s \\
0
\end{array}\right)\left(\begin{array}{c}
3 s \\
0
\end{array}\right)\left(H_{3 s}-H_{0}\right),\right. \\
& -\left(\begin{array}{c}
s \\
1
\end{array}\right)\left(\begin{array}{c}
2 s \\
1
\end{array}\right)\left(\begin{array}{c}
3 s+1 \\
1
\end{array}\right)\left(H_{3 s+1}-H_{1}\right), \ldots, \\
& \left.\left(\begin{array}{c}
s \\
s
\end{array}\right)\left(\begin{array}{c}
2 s \\
s
\end{array}\right)\left(\begin{array}{c}
3 s+s \\
s
\end{array}\right)\left(H_{3 s+s}-H_{s}\right)\right)
\end{aligned}
$$

where $a_{i}=(-1)^{i}\left(\begin{array}{c}s \\ i\end{array}\right)\left(\begin{array}{c}2 s \\ i\end{array}\right)\left(\begin{array}{c}3 s+i \\ i\end{array}\right)\left(H_{3 s+i}-H_{i}\right)$. Then there holds

$$
\left\|B_{6}\right\|_{2}=\left(\begin{array}{c}
3 s \\
s
\end{array}\right)^{2}\left(2 H_{3 s}-H_{2 s}-H_{s}\right) \text {. }
$$


Corollary 12. Let $\widetilde{B}_{5}=-B_{5}$ and $\widetilde{B}_{6}=-B_{6}$, and s is even. Then one has the identities for spectral norm

$$
\begin{gathered}
\left\|\widetilde{B}_{5}\right\|_{2}=2\left(\begin{array}{c}
2 s \\
s
\end{array}\right)^{2}\left(2 H_{2 s}-H_{s}\right), \\
\left\|\widetilde{B}_{6}\right\|_{2}=\left(\begin{array}{c}
3 s \\
s
\end{array}\right)^{2}\left(2 H_{3 s}-H_{2 s}-H_{s}\right) .
\end{gathered}
$$

\section{Spectral Norms of $g$-Circulant Matrices}

Inspired by the above propositions, we analyse spectral norms of some given $g$-circulant matrices in this section.

Lemma 13 (see [25]). The $(n+1) \times(n+1)$ matrix $Q_{g}$ is unitary if and only if

$$
(n+1, g)=1,
$$

where $Q_{g}$ is a g-circulant matrix with the first row $e^{*}=[1$, $0, \ldots, 0]$.

Lemma 14 (see [25]). A is a g-circulant matrix with the first row $\left[a_{0}, a_{1}, \ldots, a_{n}\right]$ if and only if

$$
A=Q_{g} C \text {, }
$$

where

$$
C=\operatorname{circ}\left(a_{0}, a_{1}, \ldots, a_{n}\right) .
$$

In the following part, we set $(n+1, g)=1$.

Theorem 15. Let $(n+1) \times(n+1)$-circulant matrix $B_{7}$ be as in (15), and the first row of $B_{7}$ is

$$
\begin{aligned}
& \left(\left(\begin{array}{l}
n \\
0
\end{array}\right)^{2}\left(\begin{array}{c}
2 n \\
0
\end{array}\right)\left(H_{2 n}-H_{0}\right),\right. \\
& \left(\begin{array}{c}
n \\
1
\end{array}\right)^{2}\left(\begin{array}{c}
2 n+1 \\
1
\end{array}\right)\left(H_{2 n+1}-H_{1}\right), \ldots \\
& \left.\left(\begin{array}{c}
n \\
n
\end{array}\right)^{2}\left(\begin{array}{c}
2 n+n \\
n
\end{array}\right)\left(H_{2 n+n}-H_{n}\right)\right),
\end{aligned}
$$

where $a_{i}=\left(\begin{array}{c}n \\ i\end{array}\right)^{2}\left(\begin{array}{c}2 n+i \\ i\end{array}\right)\left(H_{2 n+i}-H_{i}\right)$. Then

$$
\left\|B_{7}\right\|_{2}=2\left(\begin{array}{c}
2 n \\
n
\end{array}\right)^{2}\left(H_{2 n}-H_{n}\right) .
$$

Proof. With the help of Lemmas 13 and 14, we know that the $g$-circulant matrix $B_{7}$ is normal; then we claim that the spectral norm of $B_{7}$ is equal to its spectral radius. Furthermore, applying the irreducible and entrywise nonnegative properties, we claim that $\left\|B_{7}\right\|_{2}$ (i.e., its spectral norm) is equal to its Perron value. We select a $(n+1)$-dimensional column vector $v=(1,1, \ldots, 1)^{T}$; then

$$
B_{7} v=\left(\sum_{i=0}^{n}\left(\begin{array}{c}
n \\
i
\end{array}\right)^{2}\left(\begin{array}{c}
2 n+i \\
i
\end{array}\right)\left(H_{2 n+i}-H_{i}\right)\right) v .
$$

Obviously, $\sum_{i=0}^{n}\left(\begin{array}{c}n \\ i\end{array}\right)^{2}\left(\begin{array}{c}2 n+i \\ i\end{array}\right)\left(H_{2 n+i}-H_{i}\right)$ is an eigenvalue of $B_{7}$ associated with $v$, which is necessarily the Perron value of $B_{7}$. Employing (4), we obtain

$$
\left\|B_{7}\right\|_{2}=2\left(\begin{array}{c}
2 n \\
n
\end{array}\right)^{2}\left(H_{2 n}-H_{n}\right) .
$$

This completes the proof.

Corollary 16. Let $(n+1) \times(n+1)$-circulant matrix $B_{8}$ be as in (15), and the first row of $B_{8}$ is

$$
\begin{aligned}
& \left(\left(\begin{array}{c}
n \\
0
\end{array}\right)\left(\begin{array}{c}
2 n \\
0
\end{array}\right)\left(\begin{array}{c}
3 n \\
0
\end{array}\right)\left(H_{3 n}-H_{0}\right)\right. \\
& \left(\begin{array}{c}
n \\
1
\end{array}\right)\left(\begin{array}{c}
2 n \\
1
\end{array}\right)\left(\begin{array}{c}
3 n+1 \\
1
\end{array}\right)\left(H_{3 n+1}-H_{1}\right), \ldots \\
& \left.\left(\begin{array}{c}
n \\
n
\end{array}\right)\left(\begin{array}{c}
2 n \\
n
\end{array}\right)\left(\begin{array}{c}
4 n \\
n
\end{array}\right)\left(H_{4 n}-H_{n}\right)\right)
\end{aligned}
$$

where $a_{i}=\left(\begin{array}{c}n \\ i\end{array}\right)\left(\begin{array}{c}2 n \\ i\end{array}\right)\left(\begin{array}{c}3 n+i \\ i\end{array}\right)\left(H_{3 n+i}-H_{i}\right)$. Then one obtains

$$
\left\|B_{8}\right\|_{2}=\left(\begin{array}{c}
3 n \\
n
\end{array}\right)^{2}\left(2 H_{3 n}-H_{2 n}-H_{n}\right) .
$$

\section{Numerical Examples}

Example 1. In this example, we give the numerical results for $B_{1}$ and $B_{2}$.

Comparing the data in Table 1 , we declare that the identities of spectral norms for $B_{i}(i=1,2)$ hold.

Example 2. In this example, we list the numerical results for $B_{i}, \widetilde{B}_{i}(i=3,4)$.

With the help of data in Table 2, it is clear that the identities of spectral norms for $B_{i}, \widetilde{B}_{i}(i=3,4)$ hold.

Example 3. In this example, we reveal the numerical results for alternative skew-circulant matrices $B_{i}, \widetilde{B}_{i}(i=5,6)$.

Combining the data in Table 3 , we deduce that the identities of spectral norms for $B_{i}, \widetilde{B}_{i}(i=5,6)$ hold.

Example 4. In this example, we show numerical results for $B_{7}$ and $B_{8}$.

Considering the data in Table 4 , we deduce that the identities of spectral norms for $B_{i}(i=7,8)$ hold.

The above results demonstrate that the identities of spectral norms for the given matrices hold.

\section{Conclusion}

This paper had discussed the explicit formulae for identical estimations of spectral norms for circulant, skew-circulant and $g$-circulant matrices, whose entries are binomial coefficients combined with harmonic numbers. Furthermore, it is easy to take other entries to obtain more interesting identities, and the same approaches can be used to verify those identities. Furthermore, explicit formulas for both 
TABLE 1: Spectral norms of $B_{i}(i=1,2), C_{1, n}=2\left(\begin{array}{c}2 n \\ n\end{array}\right)^{2}\left(H_{2 n}-H_{n}\right)$, and $C_{2, n}=\left(\begin{array}{c}3 n \\ n\end{array}\right)^{2}\left(2 H_{3 n}-H_{2 n}-H_{n}\right)$.

\begin{tabular}{llcccccc}
\hline$n$ & 0 & 1 & 2 & 3 & 4 & 5 & 6 \\
\hline$\left\|B_{1}\right\|_{2}$ & 0 & 4 & 42 & $4.93 e+2$ & $6.22 e+3$ & $8.20 e+4$ & $1.12 e+6$ \\
$\left\|B_{2}\right\|_{2}$ & 0 & $1.05 e+1$ & $2.96 e+2$ & $9.69 e+3$ & $3.44 e+5$ & $1.28 e+7$ & $4.95 e+8$ \\
$C_{1, n}$ & 0 & 4 & 42 & $4.93 e+2$ & $6.22 e+3$ & $8.20 e+4$ & $1.12 e+6$ \\
$C_{2, n}$ & 0 & $1.05 e+1$ & $2.96 e+2$ & $9.69 e+3$ & $3.44 e+5$ & $1.28 e+7$ & $4.95 e+8$ \\
\hline
\end{tabular}

TABLE 2: Spectral norms of $B_{i}, \widetilde{B}_{i}(i=3,4), C_{1, m}=2\left(\begin{array}{c}2 m \\ m\end{array}\right)^{2}\left(H_{2 m}-H_{m}\right)$, and $C_{2, m}=\left(\begin{array}{c}3 m \\ m\end{array}\right)^{2}\left(2 H_{3 m}-H_{2 m}-H_{m}\right)$.

\begin{tabular}{|c|c|c|c|c|c|c|}
\hline$m$ & 1 & 3 & 5 & 7 & 9 & 11 \\
\hline$\left\|B_{3}\right\|_{2}$ & 4 & $4.93 e+2$ & $8.20 e+4$ & $1.55 e+7$ & $3.15 e+9$ & $6.68 e+11$ \\
\hline$\left\|B_{4}\right\|_{2}$ & 10.5 & $9.69 e+3$ & $1.28 e+7$ & $1.96 e+10$ & $3.20 e+13$ & $5.49 e+16$ \\
\hline$\left\|\widetilde{B}_{3}\right\|_{2}$ & 4 & $4.93 e+2$ & $8.20 e+4$ & $1.55 e+7$ & $3.15 e+9$ & $6.68 e+11$ \\
\hline$\left\|\widetilde{B}_{4}\right\|_{2}$ & 10.5 & $9.69 e+3$ & $1.28 e+7$ & $1.96 e+10$ & $3.20 e+13$ & $5.49 e+16$ \\
\hline$C_{1, m}$ & 4 & $4.93 e+2$ & $8.20 e+4$ & $1.55 e+7$ & $3.15 e+9$ & $6.68 e+11$ \\
\hline$C_{2, m}$ & 10.5 & $9.69 e+3$ & $1.28 e+7$ & $1.96 e+10$ & $3.20 e+13$ & $5.49 e+16$ \\
\hline
\end{tabular}

TABLE 3: Spectral norms of $B_{i}, \widetilde{B}_{i}(i=5,6), C_{1, s}=2\left(\begin{array}{c}2 s \\ s\end{array}\right)^{2}\left(H_{2 s}-H_{s}\right)$, and $C_{2, s}=\left(\begin{array}{c}3 s \\ s\end{array}\right)^{2}\left(2 H_{3 s}-H_{2 s}-H_{s}\right)$.

\begin{tabular}{lcccccc}
\hline$s$ & 0 & 2 & 4 & 6 & 8 & 10 \\
\hline$\left\|B_{5}\right\|_{2}$ & 0 & 42 & $6.22 e+3$ & $1.12 e+6$ & $2.20 e+8$ & $7.57 e+10$ \\
$\left\|B_{6}\right\|_{2}$ & 0 & $2.96 e+2$ & $3.44 e+5$ & $4.95 e+5$ & $1.32 e+15$ \\
$\left\|\widetilde{B}_{5}\right\|_{2}$ & 0 & 42 & $6.22 e+3$ & $1.12 e+6$ & $2.20 e+8$ & $4.57 e+10$ \\
$\left\|\widetilde{B}_{6}\right\|_{2}$ & 0 & $2.96 e+2$ & $3.44 e+5$ & $4.95 e+5$ & $7.86 e+11$ & $1.32 e+15$ \\
$C_{1, s}$ & 0 & 42 & $6.22 e+3$ & $1.12 e+6$ & $2.20 e+8$ & $4.57 e+10$ \\
$C_{2, s}$ & 0 & $2.96 e+2$ & $3.44 e+5$ & $4.95 e+5$ & $1.32 e+15$ \\
\hline
\end{tabular}

TABLE 4: Spectral norms of $B_{i}(i=7,8), C_{1, n}=2\left(\begin{array}{c}2 n \\ n\end{array}\right)^{2}\left(H_{2 n}-H_{n}\right)$, and $C_{2, n}=\left(\begin{array}{c}3 n \\ n\end{array}\right)^{2}\left(2 H_{3 n}-H_{2 n}-H_{n}\right)$.

\begin{tabular}{|c|c|c|c|c|c|c|c|c|c|}
\hline$n+1$ & & 5 & & 6 & & & 7 & & \\
\hline$g$ & 2 & 3 & 4 & 5 & 2 & 3 & 4 & 5 & 6 \\
\hline$\left\|B_{7}\right\|_{2}$ & $6.22 e+3$ & $6.22 e+3$ & $6.22 e+3$ & $8.20 e+4$ & $1.12 e+6$ & $1.12 e+6$ & $1.12 e+6$ & $1.12 e+6$ & $1.12 e+6$ \\
\hline$\left\|B_{8}\right\|_{2}$ & $3.44 e+5$ & $3.44 e+5$ & $3.44 e+5$ & $1.28 e+7$ & $4.95 e+8$ & $4.95 e+8$ & $4.95 e+8$ & $4.95 e+8$ & $4.95 e+8$ \\
\hline$C_{1, n}$ & $6.22 e+3$ & $6.22 e+3$ & $6.22 e+3$ & $8.20 e+4$ & $1.12 e+6$ & $1.12 e+6$ & $1.12 e+6$ & $1.12 e+6$ & $1.12 e+6$ \\
\hline$C_{2, n}$ & $3.44 e+5$ & $3.44 e+5$ & $3.44 e+5$ & $1.28 e+7$ & $4.95 e+8$ & $4.95 e+8$ & $4.95 e+8$ & $4.95 e+8$ & $4.95 e+8$ \\
\hline
\end{tabular}

norms $\|A\|$ and $\left\|A^{-1}\right\|$ help us to estimate the so-called condition number. It is an interesting problem to investigate the properties of $B_{i}(i=1,2, \ldots, 8)$, such as the explicit formulations for determinants and inverses, by just using the entries in the first row.

\section{Conflict of Interests}

The authors declare that there is no conflict of interests regarding the publication of this paper.

\section{Acknowledgments}

This work is supported by NFSC (no. 11201212), Promotive Research Fund for Excellent Young and Middle-Aged Scientists of Shandong Province (no. BS2012DX004), Shandong Province Higher Educational Science and Technology
Program (J13LI11), AMEP, and the Special Funds for Doctoral Authorities of Linyi University.

\section{References}

[1] W. C. Chu and L. D. Donno, "Hypergeometric series and harmonic number identities," Advances in Applied Mathematics, vol. 34, no. 1, pp. 123-137, 2005.

[2] M. H. Ang, K. T. Arasu, S. Lun Ma, and Y. Strassler, "Study of proper circulant weighing matrices with weight 9," Discrete Mathematics, vol. 308, no. 13, pp. 2802-2809, 2008.

[3] V. Brimkov, "Algorithmic and explicit determination of the Lovász number for certain circulant graphs," Discrete Applied Mathematics, vol. 155, no. 14, pp. 1812-1825, 2007.

[4] A. Cambini, "An explicit form of the inverse of a particular circulant matrix," Discrete Mathematics, vol. 48, no. 2-3, pp. 323$325,1984$. 
[5] W. S. Chou, B. S. Du, and P. J. S. Shiue, "A note on circulant transition matrices in Markov chains," Linear Algebra and Its Applications, vol. 429, no. 7, pp. 1699-1704, 2008.

[6] P. Davis, Circulant Matrices, Wiley, New York, NY, USA, 1979.

[7] C. Erbas and M. M. Tanik, "Generating solutions to the NQueens problems using 2-circulants," Mathematics Magazine, vol. 68 , no. 5, pp. 343-356, 1995.

[8] Z. L. Jiang and Z. X. Zhou, Circulant Matrices, Chengdu University of Science and Technology Press, Chengdu, China, 1999.

[9] A. Mamut, Q. X. Huang, and F. J. Liu, "Enumeration of 2regular circulant graphs and directed double networks," Discrete Applied Mathematics, vol. 157, no. 5, pp. 1024-1033, 2009.

[10] I. Stojmenović, "Multiplicative circulant networks topological properties and communication algorithms," Discrete Applied Mathematics, vol. 77, no. 3, pp. 281-305, 1997.

[11] M. Ventou and C. Rigoni, "Self-dual doubly circulant codes," Discrete Mathematics, vol. 56, no. 2-3, pp. 291-298, 1985.

[12] M. J. Weinberger and A. Lempel, "Factorization of symmetric circulant matrices in finite fields," Discrete Applied Mathematics, vol. 28, no. 3, pp. 271-285, 1990.

[13] Y. K. Wu, R. Z. Jia, and Q. Li, " $g$-circulant solutions to the $(0,1)$ matrix equation $A^{m}=J_{n}^{*}$, Linear Algebra and its Applications, vol. 345, no. 1-3, pp. 195-224, 2002.

[14] D. Bertaccini and M. K. Ng, "Skew-circulant preconditioners for systems of LMF-based ODE codes," in Numerical Analysis and Its Applications, Lecture Notes in Computer Science, pp. 93-101, 2001.

[15] R. Chan and X. Q. Jin, "Circulant and skew-circulant preconditioners for skew-hermitian type Toeplitz systems," BIT Numerical Mathematics, vol. 31, no. 4, pp. 632-646, 1991.

[16] R. Chan and M. K. Ng, "Toeplitz preconditioners for Hermitian Toeplitz systems," Linear Algebra and Its Applications C, vol. 190, pp. 181-208, 1993.

[17] T. Huclke, "Circulant and skew-circulant matrices for solving Toeplitz matrix problems," SIAM Journal on Matrix Analysis and Applications, vol. 13, no. 3, pp. 767-777, 1992.

[18] J. N. Lyness and T. SØrevik, "Four-dimensional lattice rules generated by skew-circulant matrices," Mathematics of Computation, vol. 73, no. 245, pp. 279-295, 2004.

[19] A. Bose, R. S. Hazra, and K. Saha, "Poisson convergence of eigenvalues of circulant type matrices," Extremes, vol. 14, no. 4, pp. 365-392, 2011.

[20] A. Bose, R. S. Hazra, and K. Saha, "Spectral norm of circulanttype matrices," Journal of Theoretical Probability, vol. 24, no. 2, pp. 479-516, 2011.

[21] A. Bose, S. Guha, R. S. Hazra, and K. Saha, "Circulant type matrices with heavy tailed entries," Statistics and Probability Letters, vol. 81, no. 11, pp. 1706-1716, 2011.

[22] E. Ngondiep, S. Serra-Capizzano, and D. Sesana, "Spectral features and asymptotic properties for g-circulants and gToeplitz sequences," SIAM Journal on Matrix Analysis and Applications, vol. 31, no. 4, pp. 1663-1687, 2009.

[23] S. Solak, "On the norms of circulant matrices with the Fibonacci and Lucas numbers," Applied Mathematics and Computation, vol. 160, no. 1, pp. 125-132, 2005.

[24] A. İpek, "On the spectral norms of circulant matrices with classical Fibonacci and Lucas numbers entries," Applied Mathematics and Computation, vol. 217, no. 12, pp. 6011-6012, 2011.
[25] W. T. Stallings and T. L. Boullion, "The pseudoinverse of an $r$-circulant matrix," Proceedings of the American Mathematical Society, vol. 34, no. 2, pp. 385-388, 1972.

[26] R. A. Horn and C. R. Johnson, Matrix Analysis, Cambridge University Press, Cambridge, UK, 1985 


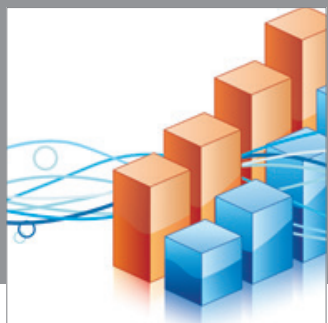

Advances in

Operations Research

mansans

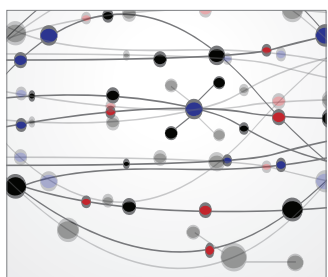

The Scientific World Journal
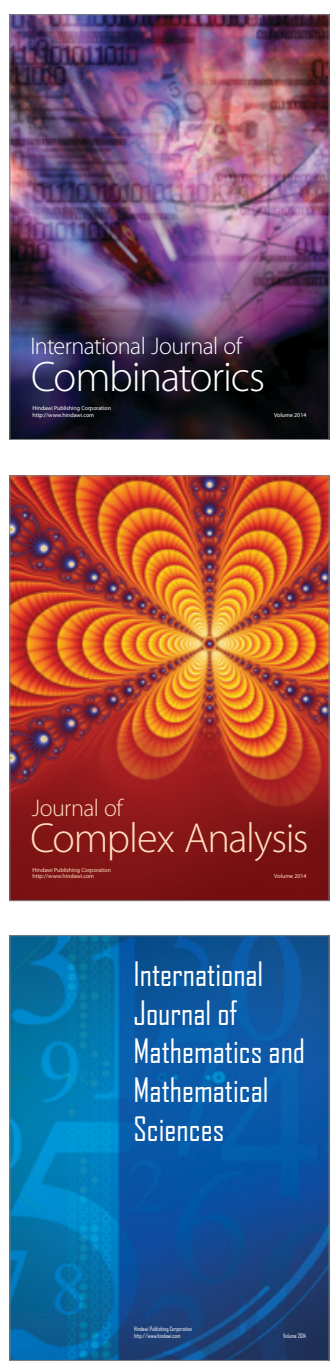
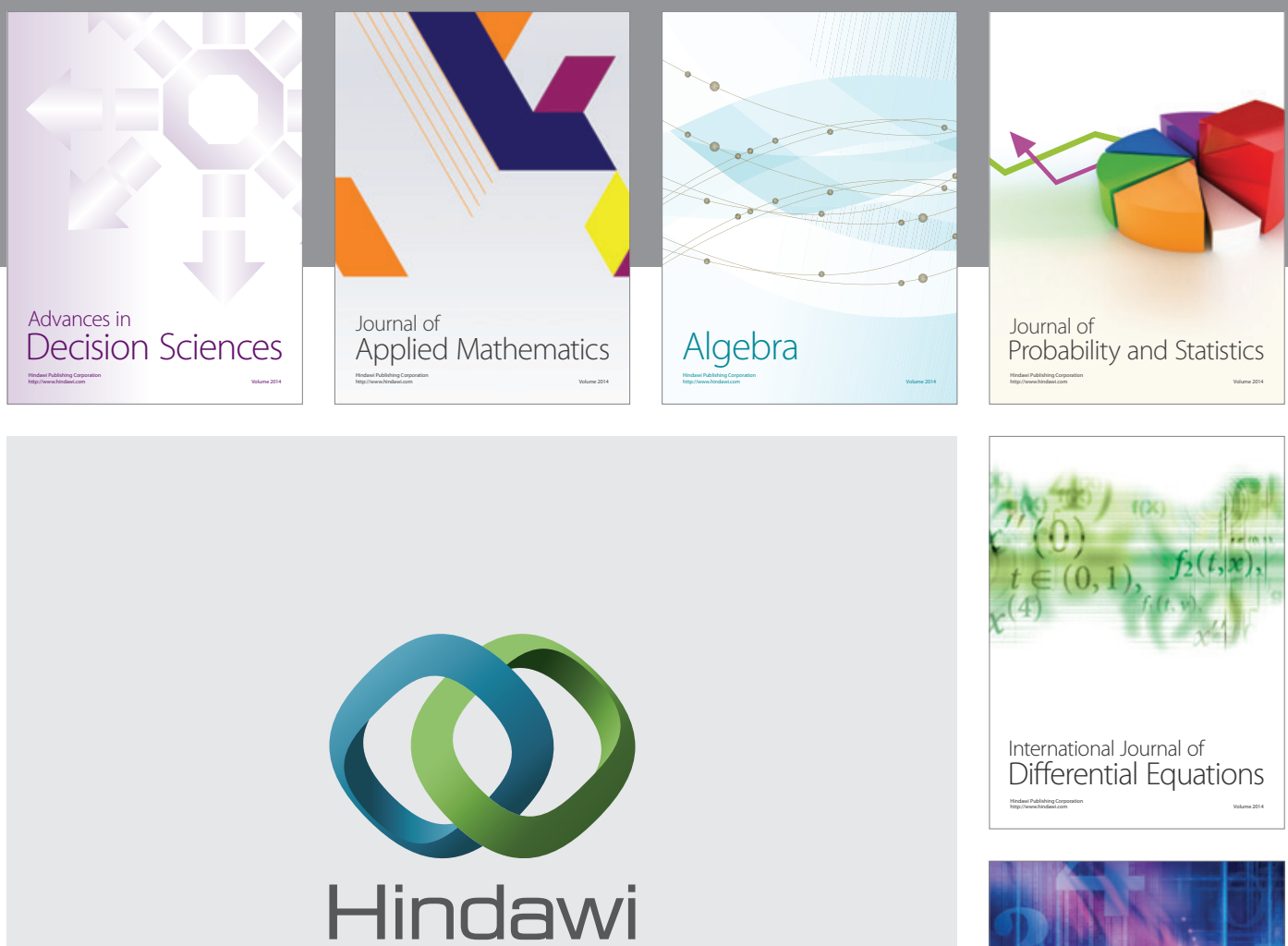

Submit your manuscripts at http://www.hindawi.com
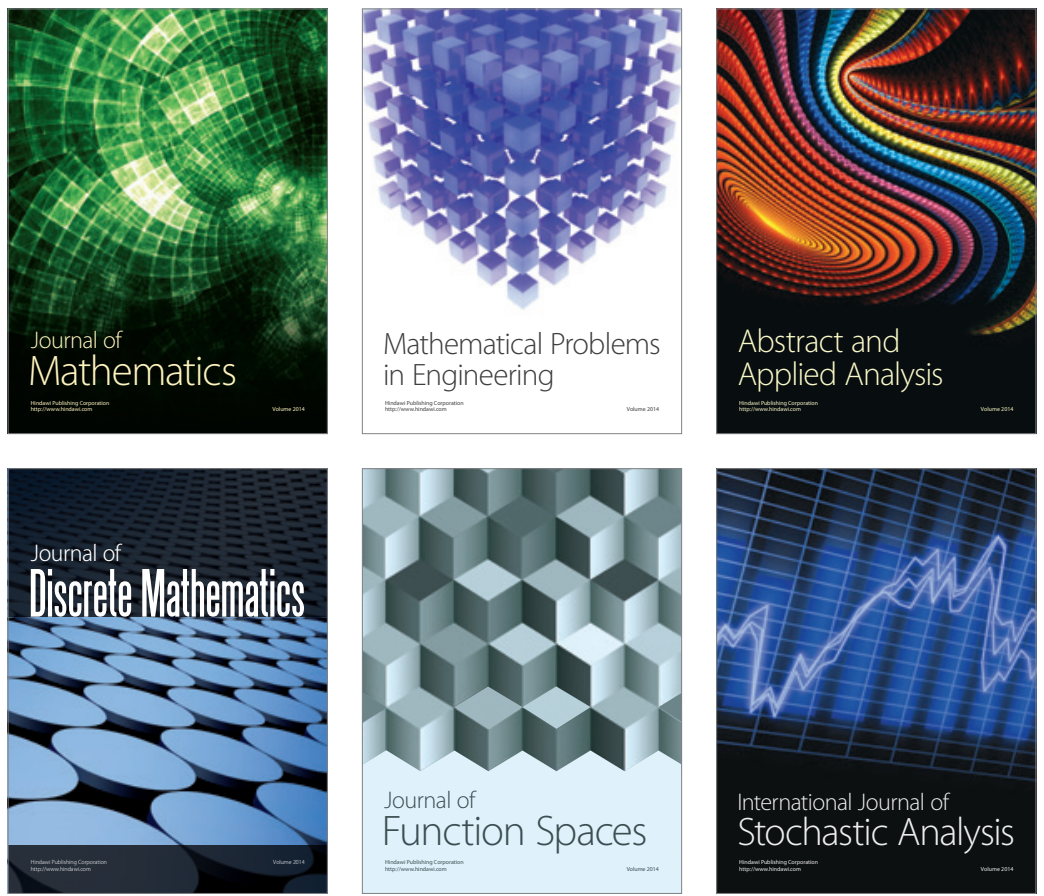

Journal of

Function Spaces

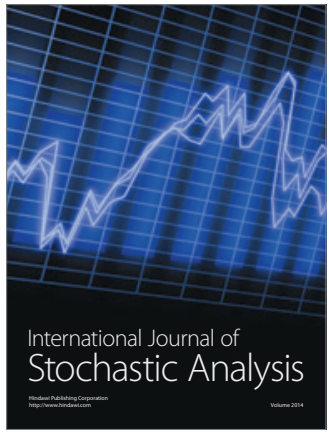

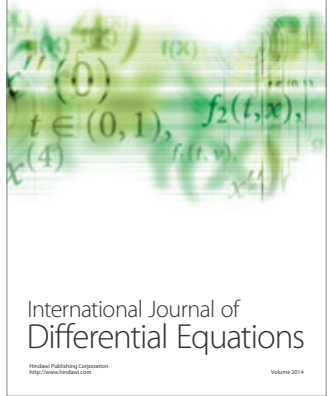
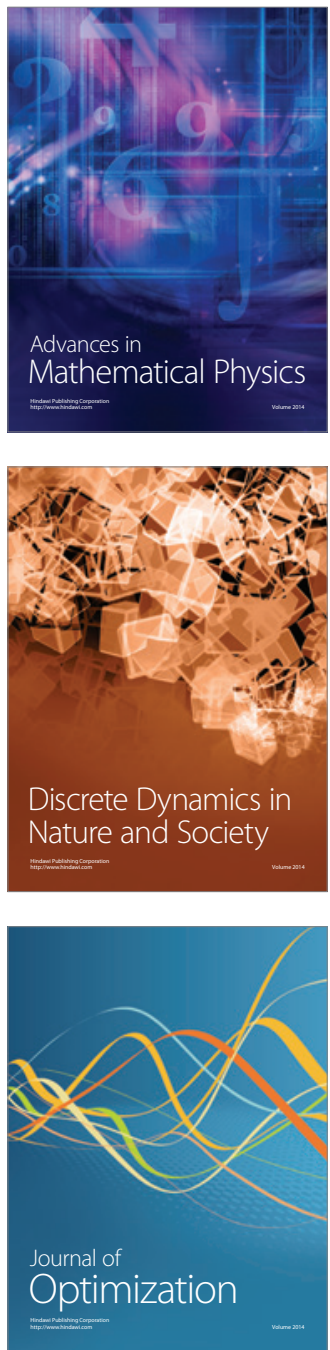